\title{
Hemodynamic effect of avanafil and glyceryl trinitrate coadministration
}

\author{
Dennis Swearingen, ${ }^{1}$ Ajay Nehra, ${ }^{2}$ Susie Morelos, ${ }^{3}$ and Craig A Peterson ${ }^{3}$ \\ 'Celerion, Tempe, AZ, USA; ${ }^{2}$ Department of Urology, Rush University Medical Center, Chicago, IL, USA; ${ }^{2}$ IVVUS, Inc, Mountain View, CA, USA
}

\begin{abstract}
A Phase I, double-blind, randomized, crossover study in healthy males ( $N=106)$ was conducted between March 21, 2004, and May 17, 2004, to determine the magnitude and duration of the hemodynamic interaction of avanafil (a phosphodiesterase type- 5 inhibitor for treating males with erectile dysfunction) when coadministered with glyceryl trinitrate (NTG) compared with sildenafil and placebo. Subjects received avanafil (200 mg), sildenafil (100 mg), and placebo (on separate days) via the oral route followed by NTG (0.4 mg) 12, 8, 4, 1, or 0.5 hours post-dose via the sublingual route. Blood pressure (BP) and heart rate (HR) were assessed at defined intervals.

Throughout the study (after administration of the study drug, and including the period after NTG administration), the effects of avanafil and sildenafil on BP and HR were significantly greatest overall, at the shortest (0.5-hour) time interval compared with placebo. By the 8- and 12-hour time intervals, no significant difference in BP or HR was observed for avanafil ( 8 and 12 hours) or sildenafil ( 12 hours) ( $p>0.05$, compared with placebo). Compared with avanafil, sildenafil had a significantly greater effect when dosed 0.5 hours before NTG on standing HR ( $p=0.05) ; 1$ hour before NTG on standing systolic blood pressure (SBP) $(p<0.05)$, sitting SBP ( $p=0.01)$ and standing HR ( $p<0.01$ ); and 12 hours before NTG on standing SBP $(p=0.05)$. Throughout the study, symptomatic hypotension adverse events occurred in $27 \%, 29 \%$, and $12 \%$, and clinically significant reductions in standing SBP ( $\geq 30 \mathrm{mmHg}$ ) occurred in $15 \%, 29 \%$, and $12 \%$ of subjects dosed with avanafil, sildenafil, and placebo, respectively (overall treatment differences: $p<0.01$ and $p<0.05$, respectively).

These data show that avanafil and sildenafil have no significant effect on BP and HR if administered to healthy males $\geq 8$ hours (avanafil) or $\geq 12$ hours (sildenafil) before a sublingual dose of NTG. However, results may differ in populations with known vascular disease, especially those using other concurrent pharmacotherapy. These findings may be of interest to clinicians who treat patients with erectile dysfunction and who also have a cardiovascular condition. Of note, the applicability of these results in such patients may be limited because the enrollment comprised healthy, normal subjects.
\end{abstract}

Keywords: avanafil, PDE5 inhibitor, erectile dysfunction, hypotension, drug-drug interaction, nitrates

Citation

Provenance

Dates

Copyright

Abbreviations

Correspondence address

Email address

Competing interests

Trial registration number
Swearingen D, Nehra A, Morelos S, Peterson CA. Hemodynamic effect of avanafil and glyceryl trinitrate coadministration. Drugs in Context 2013; 212248. doi: $10.7573 /$ dic. 212248

Submitted; externally peer reviewed

Submitted: 28 November 2012; Accepted, subject to peer review: 7 December 2012; Published: 26 February 2013

(c) 2013 Swearingen D, Nehra A, Morelos S, Peterson CA. This is an open-access article distributed under the terms of the Creative Commons Attribution License (CC-BY-NC-ND 3.0) which allows unrestricted sharing, copying and distribution for personal use provided it is properly attributed. Commercial use is not permitted.

$\mathrm{AE}$, adverse event; $\mathrm{BP}$, blood pressure; $\mathrm{Cl}$, confidence interval; $\mathrm{CVD}$, cardiovascular disease; $\mathrm{DBP}$, diastolic blood pressure; $\mathrm{ED}$, erectile dysfunction; HR, heart rate; NTG, glyceryl trinitrate; PDE, phosphodiesterase; SBP, systolic blood pressure; SD, standard deviation

Dennis Swearingen, MD, CPI, Principal Investigator, Site Medical Director, Celerion, 2420 West Baseline Road, Tempe, AZ 85283, USA

dennis.swearingen@celerion.com

Dr Swearingen and Dr Nehra have declared that there are no competing interests. Ms Morelos and Mr Peterson are employees of VIVUS, Inc.

NCT01616485, ClinicalTrials.gov

http://clinicaltrials.gov/ct2/show/NCT01616485 (sponsored by VIVUS, Inc.)

\section{Introduction}

Recent estimates suggest that erectile dysfunction (ED) affects $\approx 18$ million males in the USA [1] and $>150$ million males worldwide [2]. In general, most ED cases are considered secondary to organic disease, with $>50 \%$ of these cases being associated with vasculogenic abnormalities, including diabetes, angina, and myocardial infarction $[1,3]$.

Drugs in Context - The journal of interventions in clinical practice This full text PDF downloaded from www.drugsincontext.com Drugs in Context 2013; 212248. ISSN 1740-4398

Copyright (C) 2013 Swearingen D, Nehra A, Morelos S, Peterson CA. Distributed under the terms of the Creative Commons Attribution License CC-BY-NC-ND 3.0. No other uses without permission.
First-line pharmacologic treatment for males with various causes of ED comprises oral therapy with phosphodiesterase type-5 (PDE5) inhibitors, which have been shown to help restore penile blood flow and erections in response to sexual stimulation [4-7]. PDE5 inhibitors are predominantly metabolized by cytochrome P450 (CYP) 3A4, and drugs that inhibit CYP 3A4 can increase exposure to PDE5 inhibitors [4-7]. Therefore, with regard to concomitant use with a strong CYP 3A4 inhibitor (including ketoconazole, ritonavir, atazanavir, clarithromycin, indinavir, itraconazole, nefazodone, nelfinavir, saquinavir, and telithromycin), specific recommendations in individual package inserts for PDE5 inhibitors advise to either not use a PDE5 inhibitor concomitantly with a strong CYP 3A4 inhibitor [7] or to adjust the dosage of the PDE5 inhibitor as specified in the package insert [4-6]. For patients taking less potent inhibitors of 
CYP 3A4, such as erythromycin, the dosage of PDE5 inhibitors should be adjusted accordingly [4-7].

One of the most clinically significant safety concerns associated with PDE5 inhibitors is the potentially dangerous lowering of blood pressure (BP) that can occur if these agents are administered concurrently with organic nitrates or antihypertensive drugs [8]. Because glyceryl trinitrate (NTG) and other organic nitrates exert their hemodynamic effects by stimulating the production of cyclic guanosine monophosphate within vascular smooth muscle cells, it is reasonable to assume that concurrent use of PDE5 inhibitors (which inhibit the breakdown of cyclic guanosine monophosphate) could potentiate the hemodynamic response to nitrate administration. Furthermore, although PDE5 inhibitors have been shown to be potent inhibitors of the PDE5 isozyme, they may also weakly inhibit other PDE isozymes and affect their target tissues. PDE1, for example, may affect the contraction of vascular smooth muscle [9].

It is well known that PDE5 inhibitors, which are indicated for the treatment of ED and pulmonary arterial hypertension, are contraindicated for coadministration with nitrates [4-7, 10-11].

It is not widely known, however, whether certain pharmacologic characteristics of the PDE5 inhibitor class can reduce the potentially harmful hemodynamic effects of coadministration. This is important clinically because the prevalence of $\mathrm{ED}$ is high, particularly among older (aged $\geq 60$ years) males, and half of all patients with ED have a history of cardiovascular disease (CVD) [1].

ED has been associated with the risk factors of CVD, including hypertension, diabetes, chronic kidney disease, dyslipidemia, and obesity $[3,12-21]$. Thus, ED is also becoming recognized as an early marker of CVD [3, 22-25] and an important marker of "silent vascular disease" in males with no cardiac symptoms [26]. Data further suggest that ED, in and of itself, may also be an independent risk factor of CVD, coronary heart disease, stroke, and all-cause mortality [27].

Interestingly, data from an analysis of 9457 males in the Prostate Cancer Prevention Trial indicate that incident ED is significantly associated with the future risk of angina, myocardial infarction, and stroke [3]. It is patients with comorbid conditions, such as angina and chronic heart failure, who are most likely to require the administration of nitrates $[28,29]$. This further emphasizes the importance of studying drug-drug interactions between PDE5 inhibitors and cardiovascular pharmacotherapeutics.

Avanafil was approved by the US Food and Drug Administration for the treatment of ED in April 2012 [7]. Preclinical studies have suggested that, although this product is highly selective for PDE5, it is also highly selective against other PDE isozymes, including PDE1 $[30,31]$. Avanafil is rapidly absorbed from the gastrointestinal tract and has a relatively short half-life. Pharmacokinetic studies have shown the half-life of avanafil to be 3-5 hours, whereas it is 4 hours for sildenafil, 4-5 hours for vardenafil, and 17.5 hours for tadalafil. After oral administration, the maximum observed plasma concentration was reached within 30-45 minutes for avanafil compared with 30-120 minutes for sildenafil, 30-120 minutes for vardenafil, and 30-360 minutes for tadalafil [4-7, 32].

The Phase I study described here was conducted to determine if the high degree of specificity for PDE5 (compared with other
PDE isozymes) and/or the short half-life of avanafil affected the hemodynamic interaction with sublingual NTG compared with other PDE5-inhibitor therapies. Specifically, investigators examined the magnitude and duration of the interactions (when coadministered with NTG) of avanafil on systolic blood pressure (SBP) and HR compared with sildenafil and placebo.

\section{Methods}

This was a single-center, double-blind, randomized, three-way crossover study. Healthy male subjects with no previous exposure to nitrates were recruited from a single site.

Before study initiation, approval was obtained from the institutional review board/independent ethics committee for the study protocol. Written informed consent forms, consent-form updates, subject-recruitment procedures, and any other written information was provided to participants.

This study was conducted in full compliance with the procedures of the International Standard of Good Clinical Practice as defined by the International Conference on Harmonisation [33] and in accordance with the principles of the Declaration of Helsinki [34].

\section{Key inclusion and exclusion criteria}

The study cohort was healthy males who were 30-60 years of age and who had a resting supine BP between 100/60 $\mathrm{mmHg}$ and $150 / 95 \mathrm{mmHg}$. Subjects were excluded if they had a body mass index $>37 \mathrm{~kg} / \mathrm{m}^{2}$. The concomitant use of drugs that interfere with the metabolism of the CYP 3A4 enzyme was prohibited during the study.

\section{Treatment regimen}

Within each study group, subjects were treated in a doubleblind, three-way, crossover design. Each participant was treated (at separate visits) with placebo, avanafil, and sildenafil that were administered in random order using a Latin square design. Each subject received a single oral dose of avanafil $(200 \mathrm{mg})$, sildenafil $(100 \mathrm{mg})$, or placebo on separate days and at the specified time interval before the sublingual administration of a single NTG tablet $(0.4 \mathrm{mg})$.

\section{Timing of treatment doses}

Subjects were treated in five sequential groups (groups 1-5) that differed in the time between the administration of study drug and administration of NTG (Figure 1). All subjects received a single oral dose of two avanafil 100-mg capsules, two sildenafil 50-mg capsules, or two placebo capsules. Participants were challenged with one Nitrostat ${ }^{\circledR}$ (nitroglycerin tablet; $0.4 \mathrm{mg} ; 1 / 150$ grain; United States Pharmacopeia; Pfizer Inc: New York, NY, USA) administered via the sublingual route 12 hours (group 1), 8 hours (group 2), 4 hours (group 3), 1 hour (group 4), or 0.5 hours (group 5) after the administration of study medication. Subjects were assigned to study groups sequentially, beginning with group 1 (12 hours) (see Figure 1). 
Figure 1. Schedule of dosing visits and assessments. NTG, glyceryl trinitrate.

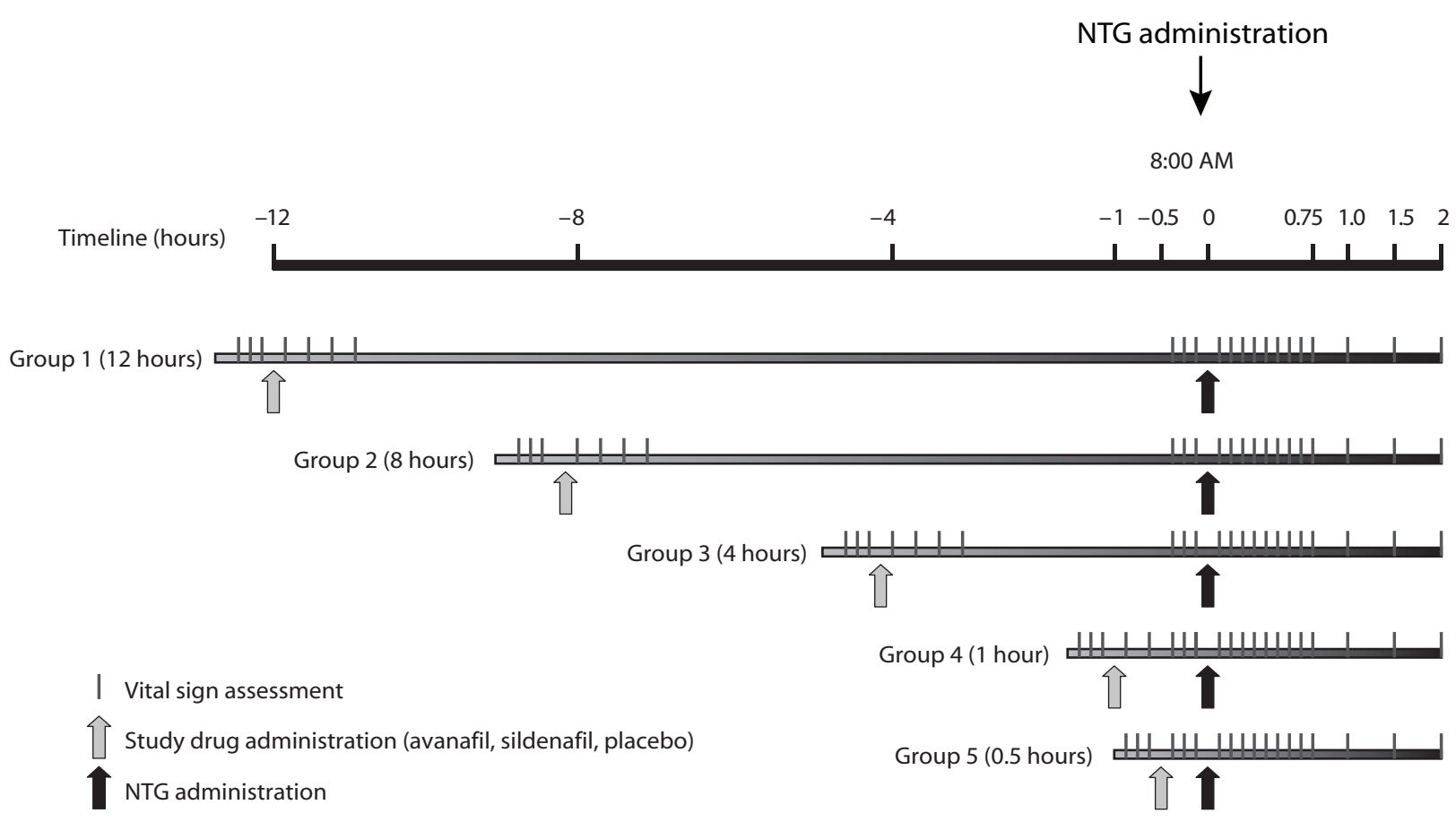

doi: 10.7573/dic.212248.f001

\section{Primary variables}

The primary hemodynamic variables were BP and HR, which were assessed while subjects were in seated and standing positions before dosing and at specified time intervals after NTG administration.

\section{Efficacy and safety monitoring}

Assessments of hemodynamics and safety were obtained throughout the study. BP and HR (standing and sitting for both measurements) were monitored and recorded at each study visit in which drugs were administered. Adverse events (AEs) were evaluated throughout the study. Electrocardiography was administered at screening and during each dosing period. Physical examinations and laboratory analyses (including complete blood count with differential, chemistry panel, and urinalyses with microscopic evaluation) were evaluated at screening and study exit. Eligibility criteria were reviewed and confirmed at screening and during each dosing period.

\section{Monitoring of hemodynamic variables}

Within each study group, we evaluated (i) the maximum change (post-dose maximum decrease in BP or maximum increase in HR) from pre-dose hemodynamic values; (ii) the mean change in these values from pre-dose across all post-dose time points; and (iii) the proportion of subjects with clinically significant decreases in BP (a decrease in SBP $>30 \mathrm{mmHg}$, a decrease in diastolic blood pressure [DBP] of $>20 \mathrm{mmHg}$, or symptomatic hypotension AEs).
Appropriate supportive-care measures were utilized for subjects who had clinically significant decreases in BP or symptomatic hypotension AEs. These included palpitations, tachycardia, visual disturbance, blurred vision, nausea, vomiting, dizziness, syncope (defined as loss of consciousness), hypotension, and pallor.

\section{Statistical evaluations}

Mean maximum hemodynamic (BP and HR) changes from baseline according to study group were calculated using the mean of three pre-dose baseline measurements obtained 15, 10, and 5 minutes before the administration of the study drug or NTG. This led to the generation of two types of pre-dose baselines. The primary focus of the present study was the period after the administration of NTG when the administration of avanafil or sildenafil might potentiate the pressure-lowering effects of NTG.

For statistical evaluation of the maximum and mean hemodynamic changes, pairwise comparisons of the active treatments (avanafil or sildenafil) with placebo were evaluated by the Wilcoxon signed-rank test. The same test was also used to compare avanafil with sildenafil.

The overall statistical significance of the hemodynamic differences among treatments with the three study drugs was evaluated by the Friedman test. Nonparametric tests using the KruskalWallis test were used to evaluate overall differences among the five groups as determined by the time intervals between the administration of the study drug and of NTG. 


\section{Results}

\section{Subject disposition}

A total of 106 males were enrolled, and 88 of these completed all three treatments. All of the subjects were included in the hemodynamic (BP and HR) and safety analyses. Of the eighteen subjects who did not complete all three treatments, two subjects withdrew from the study voluntarily, nine were withdrawn by the principal investigator for safety concerns, six were lost to follow-up, and one did not meet the entry criteria (failed a drug/alcohol screening test).

\section{Demographics and baseline characteristics}

Baseline characteristics between the study groups were similar (Table 1). The pre-dose sitting and standing SBP and DBP, as well as the respective HRs, were comparable among all groups for all periods.

\section{Safety and efficacy}

Analyses of hemodynamics before NTG administration. The maximum changes in BP and HR after the administration of the study drug (avanafil, sildenafil, or placebo) until the administration of NTG were analyzed. These results represent changes in vital signs that result from the study drugs alone (without NTG).

When data from all the groups (1 to 5) were combined, the maximum mean sitting and standing DBP decreases were signifcantly greater $(p<0.01)$ for avanafil and sildenafil compared with placebo (Table 2). The actual DBP decreases from pre-dose, however, remained $<7 \mathrm{mmHg}$ for avanafil, sildenafil, and placebo. The maximum mean sitting (after administration of sildenafil) and standing (after administration of avanafil and sildenafil) SBP decreases were significantly greater than those for placebo $(p=0.05)$, even though the decreases were $<9 \mathrm{mmHg}$. For maximum mean sitting increases in HR, sildenafil treatment exhibited a highly significant $(p<0.01)$ increase from pre-dose compared with placebo, whereas the change in mean sitting HR for avanafil treatment was not significantly different from placebo $(p>0.05)$. There were no significant differences, overall, between avanafil and sildenafil regarding changes in SBP, DBP, or HR $(p>0.05)$.

Mean maximum hemodynamic changes throughout the study. Maximum (placebo-subtracted) changes in BP and HR after administration of the study drug throughout the study (including the period after the administration of NTG) are illustrated in Figure 2. These results represent changes in vital signs that were due to the administration of the study drugs plus NTG.

Overall, the hemodynamic effects of treatment with avanafil and sildenafil decreased significantly as they were dosed further apart from NTG administration (see Figure 2). Compared with placebo, avanafil and sildenafil had no significant effects on BP if administered 8 hours or 12 hours before NTG $(p>0.05)$. Avanafil and sildenafil also had no significant effects on HR (sitting and standing, overall) when administered 4,8 , or 12 hours before NTG (avanafil) or 12 hours before NTG (sildenafil) $(p>0.05)$.

When NTG was dosed 0.5 hours after administration of the study drug, however, the mean maximum decrease in sitting SBP, and sitting and standing DBP, was significantly greater for avanafil and sildenafil relative to placebo $(p<0.05)$. For standing SBP, mean maximum decreases from placebo were significantly greater for avanafil $(p<0.05)$ but not for sildenafil $(p>0.05)$

Table 1. Baseline demographics, overall and by group.

\begin{tabular}{|c|c|c|c|c|c|c|}
\hline Parameter & $\begin{array}{c}\text { Group } 1 \text { (12 hours) } \\
(n=16)\end{array}$ & $\begin{array}{c}\text { Group } 2 \text { ( } 8 \text { hours) } \\
(n=16)\end{array}$ & $\begin{array}{c}\text { Group } 3 \text { (4 hours) } \\
(n=26)\end{array}$ & $\begin{array}{c}\text { Group } 4 \text { (1 hour) } \\
(n=24)\end{array}$ & $\begin{array}{c}\text { Group } 5 \text { (0.5 hours) } \\
(n=24)\end{array}$ & $\begin{array}{c}\text { Total } \\
(\mathrm{N}=106)\end{array}$ \\
\hline \multicolumn{7}{|l|}{ Race, n (\%) } \\
\hline Caucasian & $8(50)$ & $9(56)$ & $14(54)$ & $16(67)$ & $8(33)$ & $55(52)$ \\
\hline Hispanic & $8(50)$ & $4(25)$ & $10(39)$ & $5(21)$ & $15(63)$ & $42(40)$ \\
\hline Black & $0(0)$ & $3(19)$ & $2(8)$ & $3(13)$ & $0(0)$ & $8(8)$ \\
\hline Asian & $0(0)$ & $0(0)$ & $0(0)$ & $0(0)$ & $1(4)$ & $1(1)$ \\
\hline Age, years, mean (SD) & $43(8.98)$ & $43(8.94)$ & $43(9.13)$ & $46(9.03)$ & $42(9.17)$ & $43(9.01)$ \\
\hline Weight, Ib, mean (SD) & $180(42.3)$ & $191(33.2)$ & $185(30.2)$ & 199 (34.8) & $177(23.3)$ & $187(32.9)$ \\
\hline Height, in, mean (SD) & $69(3.4)$ & $70(2.8)$ & $68(3.4)$ & $70(3.0)$ & $67(2.7)$ & $69(3.2)$ \\
\hline
\end{tabular}

\section{Abbreviations}

SD, standard deviation

doi: $10.7573 /$ dic. $212248 . t 001$

Table 2. Mean maximum change in blood pressure and heart rate before administration of glyceryl trinitrate, all patient groups (1-5) combined.

\begin{tabular}{|c|c|c|c|c|c|c|}
\hline \multirow[t]{2}{*}{ Group } & \multicolumn{3}{|c|}{ Sitting } & \multicolumn{3}{|c|}{ Standing } \\
\hline & Avanafil & Sildenafil & Placebo & Avanafil & Sildenafil & Placebo \\
\hline $\mathrm{SBP}, \mathrm{mmHg}$ & $-6.79^{a, b}$ & $-7.39^{c}$ & -5.35 & $-8.61^{b, c}$ & $-7.88^{b}$ & -5.70 \\
\hline $\mathrm{DPB}, \mathrm{mmHg}$ & $-6.07^{b, d}$ & $-6.32^{d}$ & -3.51 & $-6.55^{b, d}$ & $-6.53^{c, d}$ & -3.90 \\
\hline Pulse, bpm & $3.47^{a, b}$ & $4.19^{d}$ & 1.67 & $4.21^{a, b}$ & $5.12^{\mathrm{a}}$ & 4.01 \\
\hline
\end{tabular}

${ }^{\mathrm{a}} p>0.05$, compared with placebo; ${ }^{\mathrm{b}} p>0.05$, compared with sildenafil; ${ }^{\mathrm{c}} p=0.05$, compared with placebo; ${ }^{\mathrm{d}} p<0.01$, compared with placebo. 
Figure 2. Placebo-subtracted, mean maximum change $(90 \% \mathrm{Cl})$ in blood pressure and heart rate, by study group throughout the study: 12 hours (group 1), 8 hours (group 2), 4 hours (group 3), 1 hour (group 4), and 0.5 hours (group 5 ).

A.

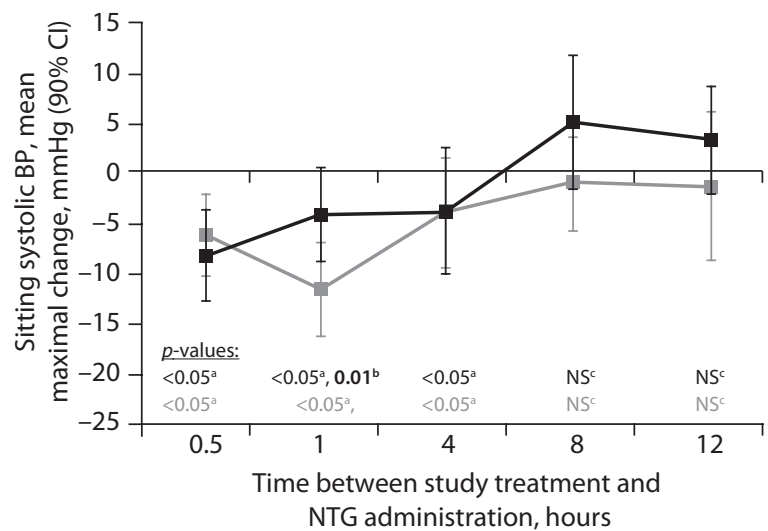

C.

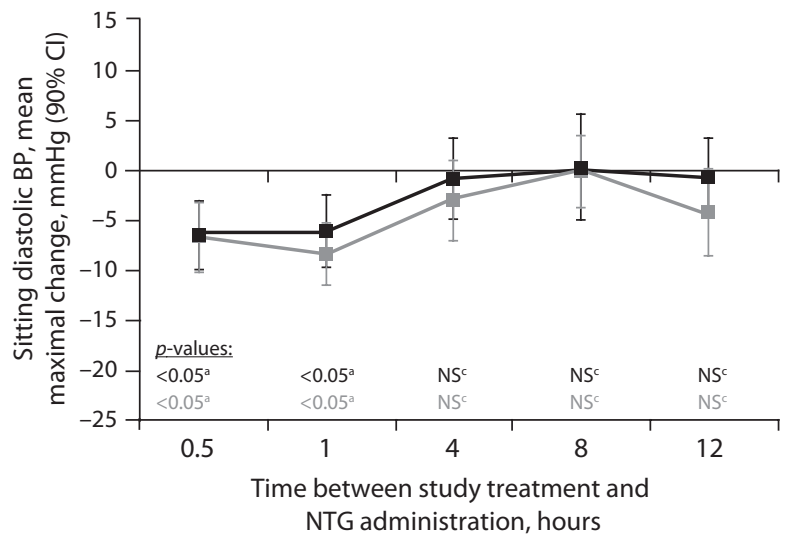

E.

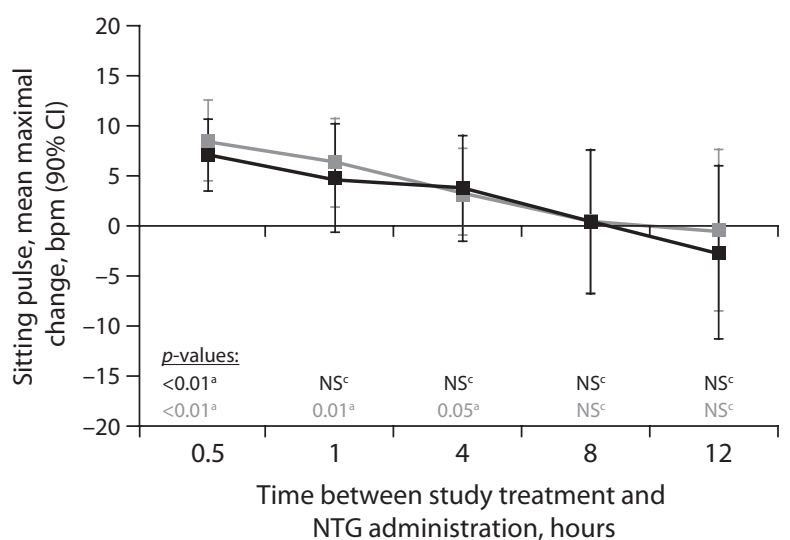

B. Standing Systolic BP (mmHg) $\quad \longrightarrow$ Sildenafil

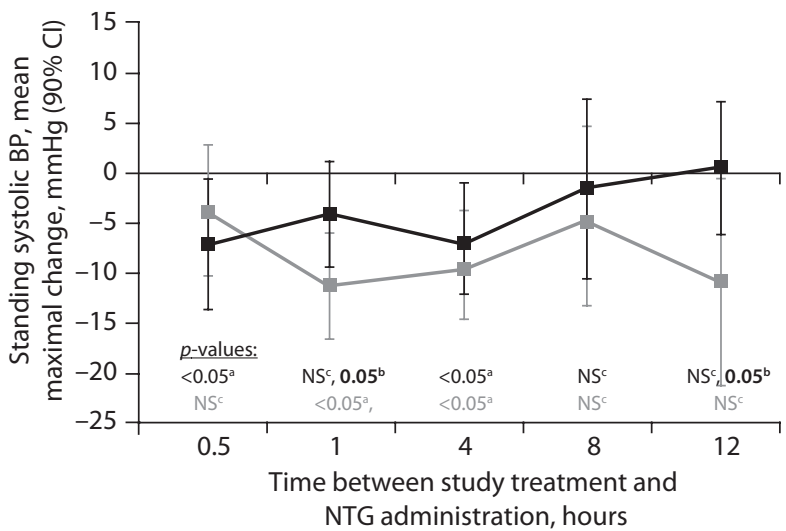

D. Standing Diastolic BP (mmHg) Sildenafil

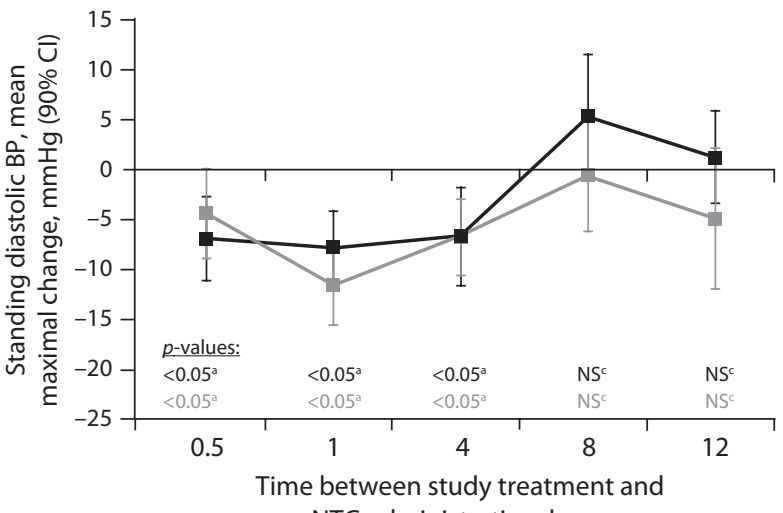
NTG administration, hours

F. Standing Pulse (bpm) $\longrightarrow$ Avanafil
$\longrightarrow$ Sildenafil

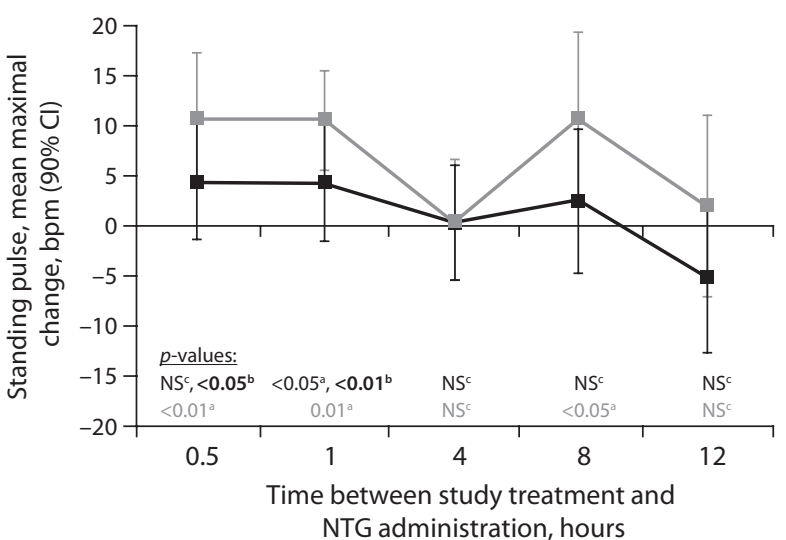

aSignificant difference from placebo; ${ }^{b}$ Significant difference from sildenafil ( $p$-values in bold type); ${ }^{c}$ No difference from placebo $(p>0.05)$. Abbreviations $\mathrm{BP}$, blood pressure; $\mathrm{Cl}$, confidence interval; NTG; glyceryl trinitrate doi: $10.7573 /$ dic.212248.f002 
Table 3. Adverse events reported by $\geq 3 \%$ of subjects, overall, and before and after NTG administration (safety population), n (\%).

\begin{tabular}{lccc}
\hline Adverse event & $\begin{array}{c}\text { Avanafil } \\
(\mathbf{n}=102)\end{array}$ & $\begin{array}{c}\text { Sildenafil } \\
(\mathbf{n}=\mathbf{9 7})\end{array}$ & $\begin{array}{c}\text { Placebo } \\
(\mathbf{n}=97)\end{array}$ \\
$\begin{array}{l}\text { Before NTG administration } \\
\text { Subjects with any AE }\end{array}$ & $16(16)$ & $8(8)$ & $4(4)$ \\
Headache & $12(12)$ & $3(3)$ & $3(3)$ \\
Dizziness & $3(3)$ & $3(3)$ & $0(0)$ \\
Nausea & $3(3)$ & $1(1)$ & $0(0)$ \\
After NTG administration & & & \\
Subjects with any AE & $42(41)$ & $43(44)$ & $28(29)$ \\
Headache & $24(24)$ & $25(26)$ & $16(16)$ \\
Dizziness & $18(18)$ & $22(23)$ & $10(10)$ \\
Nausea & $10(10)$ & $9(9)$ & $1(1)$ \\
Pallor & $9(9)$ & $8(8)$ & $4(4)$ \\
Hyperhidrosis & $6(6)$ & $7(7)$ & $1(1)$ \\
Chest discomfort & $3(3)$ & $4(4)$ & $0(0)$ \\
Hypotension & $1(1)$ & $5(5)$ & $0(0)$ \\
Vomiting & $3(3)$ & $2(2)$ & $1(1)$ \\
Syncope & $4(4)$ & $1(1)$ & $0(0)$ \\
Flushing & $1(1)$ & $3(3)$ & $0(0)$ \\
\hline
\end{tabular}

\section{Abbreviations}

$\mathrm{AE}$, adverse event; NTG, glyceryl trinitrate doi: 10.7573/dic.212248.t003

(see Figure 2). The mean maximum increase in HR was also significantly greater for avanafil and sildenafil (sitting heart rate; $p<0.01$ for both) and sildenafil (standing HR; $p<0.01$ ) compared with placebo.

As further illustrated in Figure 2, sildenafil had a significantly greater effect compared with avanafil when dosed 0.5 hours before NTG on standing HR $(p=0.05)$ : 1 hour before NTG on standing SBP $(p<0.05)$, sitting SBP $(p=0.01)$ and standing HR $(p<0.01)$; and 12 hours before NTG on standing SBP $(p=0.05)$.

Safety. Eighty-six subjects received all study drugs according to the protocol. No deaths or other serious AEs were reported, and there were no instances of priapism.
Discontinuations. Eight subjects left the study because of AEs. The AEs (mild to moderate in severity) were headache $(n=2)$, hypotension $(n=2)$, dizziness, musculoskeletal stiffness, prolonged QTc interval, and vomiting ( $\mathrm{n}=1$, each). Of these AEs, hypotension, headache, and dizziness were considered likely to be related to the study drug.

Reported AEs. Overall, 291 AEs were reported during the study. All AEs were mild or moderate in severity; no severe AEs or serious AEs were reported. Most AEs, in all treatment groups, were reported after the administration of NTG. Headache and dizziness were the most frequently reported before and after NTG administration (Table 3). These were most frequently observed in groups 3 ( 4 hours), 4 (1 hour), and 5 (0.5 hours).

Syncope, which was reported by four subjects (4\%) following avanafil dosing, and by one subject (1\%) following dosing with sildenafil (Table 3), was considered by the investigator to be moderate in severity for two subjects in the avanafil group and for the one subject in the sildenafil group. All events were considered to be related to the study drug (Table 4), occurred 7-8 minutes following administration of NTG, and resolved within 1-2 minutes. The only supportive measures required were to keep subjects in the recumbent position.

Most of the AEs reported after administration of NTG were considered to be related to the study drug regardless of the timing of NTG administration; only 12 events were considered to be unrelated to treatment. The most frequently reported treatment-related AEs were headache, dizziness, nausea, and pallor. After the administration of the study drug but before NTG treatment, only two AEs (headache: one each in the avanafil and sildenafil groups) were classified as being unrelated to treatment.

Symptomatic hypotension AEs. Throughout the study (starting with pretreatment with the study drug and after the administration of NTG), symptomatic hypotension AEs were reported in $27 \%, 29 \%$, and $12 \%$ of subjects treated with avanafil,

Table 4. All subjects experiencing syncope following NTG administration.

\begin{tabular}{|c|c|c|c|c|c|c|c|}
\hline \multirow[t]{2}{*}{$\begin{array}{l}\text { Study } \\
\text { subject }\end{array}$} & \multirow[t]{2}{*}{ Treatment } & \multirow[t]{2}{*}{ Group } & \multicolumn{3}{|c|}{ Syncope onset, severity and resolution ${ }^{a}$} & \multicolumn{2}{|c|}{$\begin{array}{l}\text { Vital signs (taken approximately } \\
3 \text { minutes from syncope onset) }\end{array}$} \\
\hline & & & $\begin{array}{l}\text { Time from NTG } \\
\text { administration to onset }\end{array}$ & Severity & $\begin{array}{l}\text { Time to } \\
\text { resolution }\end{array}$ & $\begin{array}{c}\text { Blood } \\
\text { pressure }\end{array}$ & $\begin{array}{l}\text { Pulse, } \\
\text { BPM }\end{array}$ \\
\hline 1 & Avanafil & $\begin{array}{c}\text { Group } 5 \\
(0.5 \text { hours })\end{array}$ & 8 minutes & Mild & 2 minutes & $97 / 53$ & 71 \\
\hline 2 & Avanafil & $\begin{array}{l}\text { Group } 5 \\
(0.5 \text { hours })\end{array}$ & 8 minutes & Moderate & $<1$ minute & $106 / 61$ & 44 \\
\hline 3 & Avanafil & $\begin{array}{l}\text { Group } 5 \\
\text { (0.5 hours) }\end{array}$ & 7 minutes & Mild & 2 minutes & $113 / 56$ & 60 \\
\hline 4 & Avanafil & $\begin{array}{l}\text { Group } 3 \\
\text { (4 hours) }\end{array}$ & 8 minutes & Moderate & 2 minutes & $90 / 55$ & 74 \\
\hline 5 & Sildenafil & $\begin{array}{l}\text { Group } 3 \\
\text { (4 hours) }\end{array}$ & 8 minutes & Moderate & 1 minute & $60 / 40$ & $44^{b}$ \\
\hline
\end{tabular}

${ }^{a}$ All episodes of syncope were considered to be related to study drug; ${ }^{\text {b }}$ Pulse measured 3 minutes following the blood pressure (60/40) measurement. Abbreviations

BPM, beats per minute; ND, no data available

doi: $10.7573 /$ dic.212248.t004 
Table 5. Subjects with symptomatic hypotension adverse events throughout the study. ${ }^{\mathrm{a}}$

\begin{tabular}{lcccc}
\hline Group ${ }^{\mathrm{b}} \mathrm{n}(\%)$ & \multicolumn{3}{c}{$\begin{array}{c}\text { Subjects with symptomatic } \\
\text { hypotension adverse events }\end{array}$} & $p^{\mathrm{d}}$ \\
\cline { 2 - 5 } & Avanafil & Sildenafil & Placebo $^{\mathrm{d}}$ & \\
\hline 1 (12 hours) & $3(19)$ & $5(36)$ & $2(14)$ & $>0.05$ \\
2 (8 hours) & $3(20)$ & $3(20)$ & $0(0)$ & $\mathrm{N} / \mathrm{A}^{\mathrm{e}}$ \\
3 (4 hours) & $6(25)$ & $8(36)$ & $2(9)$ & $\mathrm{N} / \mathrm{A}^{\mathrm{e}}$ \\
$4(1$ hour) & $7(30)$ & $6(26)$ & $3(14)$ & $>0.05$ \\
5 (0.5 hours) & $9(38)$ & $6(26)$ & $5(21)$ & $>0.05$ \\
1 to 5 combined & $28(27)$ & $28(29)$ & $12(12)$ & $<0.01$ \\
\hline
\end{tabular}

${ }^{\mathrm{N}} \mathrm{N} / \mathrm{A}$, not applicable.

${ }^{b} \mathrm{n}=14$ to $16,15,22$ to 23,21 to 23 , and 23 to 24 for groups $1,2,3,4$, and 5, respectively.

'Adverse events that constitute symptomatic hypotension are palpitations, tachycardia, visual disturbance, blurred vision, nausea, vomiting, dizziness, syncope, hypotension, and pallor.

dThe $p$ value is from a repeated-measures analysis on frequency data for overall treatment differences.

${ }^{\mathrm{e} A} p$ value cannot be calculated for those cases with sampling zero (subjects with missing treatments are presented but excluded from the statistical analysis).

doi: $10.7573 /$ dic.212248.t005

sildenafil, and placebo, respectively (Table 5). At the 0.5-hour dosing interval, however, when plasma levels of avanafil are expected to peak, the prevalence of symptomatic hypotension AEs was increased in patients treated with avanafil compared with those treated with sildenafil ( $38 \%$ vs. $26 \%$ ), but differences in overall treatment were not significant $(p>0.05)$. At the 4-, 8-, and 12-hour intervals, the prevalence of symptomatic hypotension AEs was significantly higher with sildenafil compared with avanafil $(36 \%$ vs. $19 \%$ at the 12 -hour interval; $p<0.05)$. Overall, symptomatic hypotension AEs occurred more frequently with avanafil and sildenafil than with placebo $(p<0.01)$.

After the administration of NTG, the only significant difference in the proportion of subjects with clinically significant decreases in DBP $(>20 \mathrm{mmHg})$ was standing BP for group 5 (at 0.5 hours) $(p<0.05): 54 \%(\mathrm{n} / \mathrm{N}=13 / 24), 48 \%(\mathrm{n} / \mathrm{N}=11 / 23)$, and $25 \%(\mathrm{n} / \mathrm{N}=6 / 24)$ for avanafil, sildenafil, and placebo groups, respectively (overall treatment difference, $p<0.05$ ).

The number of subjects with a clinically significant drop in standing SBP ( $\geq 30 \mathrm{mmHg}$ ), overall, after NTG administration was 15/100 (15\%), 28/97 (29\%), and 11/92 (12\%) for avanafil, sildenafil, and placebo groups, respectively (Figure 3). For individual groups, clinically significant decreases in standing SBP were observed only for group 4 (at 1 hour) $(p<0.05)$ and for all groups combined $(p<0.05)$.

Laboratory values. No abnormalities in hematology, serum chemistry or urinalysis were classified as AEs during the study. Mean results for hematology and mean serum chemistry were similar at screening and study exit. The mean values also remained within their respective reference ranges for all groups.

Figure 3. Percentage of subjects with a clinically significant change ( $\geq 30 \mathrm{mmHg}$ decrease) in standing systolic blood pressure from administration of study drug to post-NTG administration. ${ }^{2}$

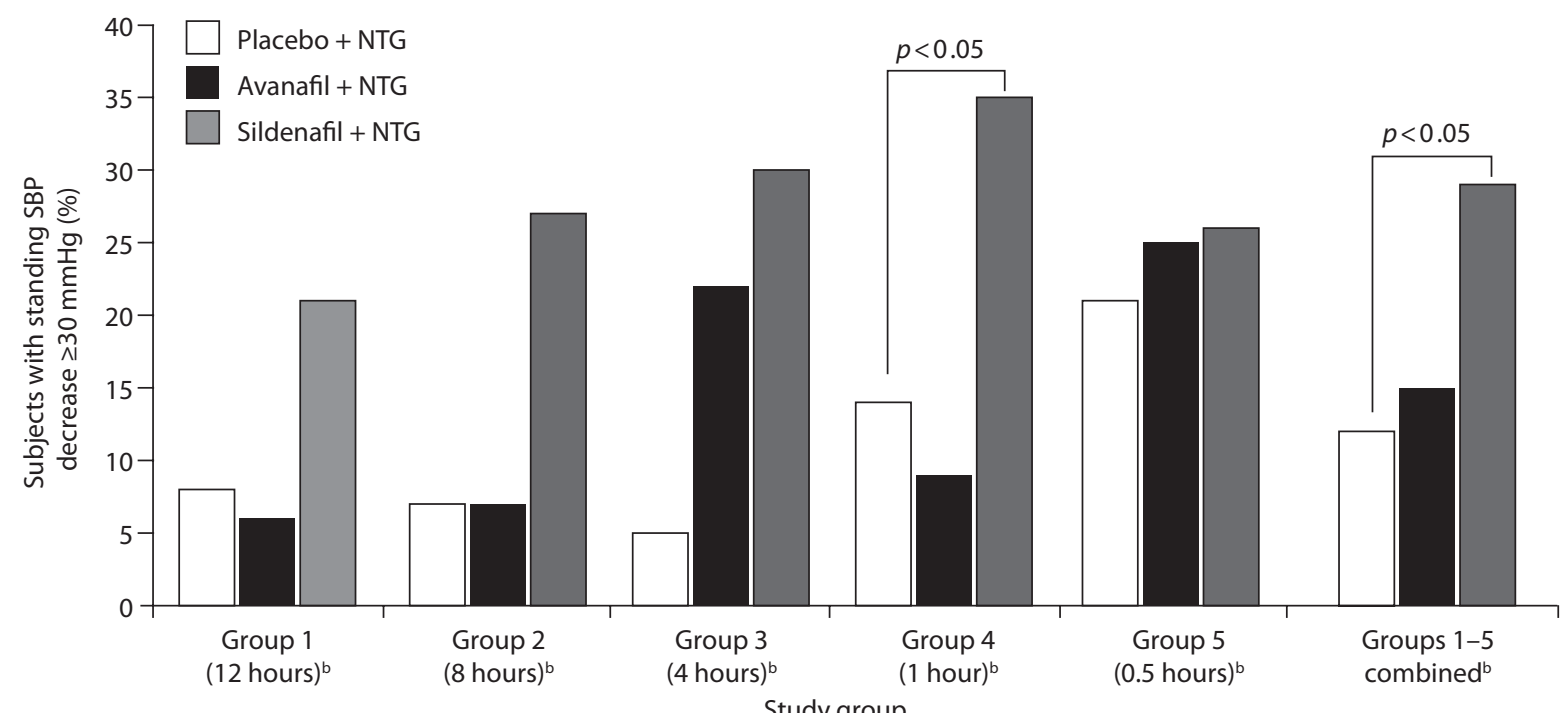

(Time interval between study drug and NTG administration)

${ }^{a} p$-values cannot be calculated for those cases with sampling zero (subjects with missing treatments are presented but are excluded from the statistical analysis).

bStudy group represents the time interval between study drug (avanafil, sildenafil, and placebo) and NTG administration.

Abbreviations

NTG, glyceryl trinitrate; SBP, systolic blood pressure

doi: $10.7573 /$ dic. $212248 . f 003$ 


\section{Discussion}

When a standard dose of sublingual NTG was administered to healthy male subjects at intervals ranging from 0.5 hours to 12 hours after a single dose of avanafil, sildenafil, or placebo, the effects of avanafil and sildenafil on BP and HR were significantly greater at the shorter (i.e., 0.5-, 1- and 4-hour) time intervals, overall. Hemodynamic interactions between avanafil or sildenafil and NTG were strongest when avanafil and sildenafil were dosed 0.5 hours before the administration of NTG (significant differences from placebo for all hemodynamic parameters except for standing HR for avanafil and standing SBP for sildenafil). As the time between the administration of avanafil or sildenafil and NTG increased, the effects on BP and HR became less pronounced. Ultimately, the hemodynamic (BP and HR) interaction compared with placebo was not significant when the time between the administration of avanafil and NTG reached 8 hours for avanafil and 12 hours for sildenafil $(p>0.05)$.

Overall, compared with sildenafil, avanafil resulted in numerically smaller changes in SBP and HR throughout the study when administered concomitantly with NTG (Figure 2). These changes were significant, however, only if avanafil and sildenafil were dosed 0.5 hours before NTG (for standing pulse), 1 hour before NTG (for sitting and standing SBP and standing pulse), and 12 hours before NTG (for standing SBP).

Throughout the study, significantly fewer subjects, overall, developed clinically significant reductions $(\geq 30 \mathrm{mmHg})$ in SBP with avanafil compared with sildenafil $(p<0.05)$. This may (at least in part) reflect the relatively shorter half-life of avanafil (which may reduce the duration of the hemodynamic interaction with NTG compared with sildenafil). It may also reflect the relatively weaker inhibition of PDE1 by avanafil. In vitro data show that the half-maximal inhibitory concentration $\left(\mathrm{IC}_{50}\right)$ of avanafil toward PDE1 is 53,000 compared with an $\mathrm{IC}_{50}$ of 600 for sildenafil (88-fold difference) [30].

Although fewer subjects who were treated with avanafil (compared with sildenafil) had decreases of $\geq 30 \mathrm{mmHg}$ in SBP, the number of patients who reported symptomatic hypotension AEs was similarly increased over placebo upon treatment with avanafil and sildenafil. After the administration of NTG, symptomatic hypotension was observed more frequently in active treatments ( $24 \%$ for avanafil and $27 \%$ for sildenafil, overall) compared with placebo (11\%) (overall treatment differences: $p<0.05$ ).

A further observation is that none of the subjects with syncope had heart rates that exceeded 74 beats per minute in proximity to their syncopal event. This suggests some level of vasovagal response that may have contributed to the event. It further suggests that patients who may be taking a concomitant drug that prevents the compensatory increase in heart rate that typically occurs when blood pressure is lowered acutely may be more likely to exhibit potentially serious syncopal events as a result of receiving such a combination.

Overall, these data show that avanafil or sildenafil administered to healthy males $\geq 8$ hours (avanafil) and $\geq 12$ hours (sildenafil) before a sublingual dose of NTG did not have a significant effect on BP and HR. These findings may be of relevance to healthcare providers who treat patients with ED who may also be at risk for myocardial infarction (and who may require on-de- mand nitrates) as well as emergency medical personnel responding to a patient who may have taken a PDE5 inhibitor.

Current US guidelines for patients with stable ischemic heart disease recommend strict avoidance of concomitant use of PDE5 inhibitors within 24 hours of nitrate administration due to the risk of profound hypotension (decrease in SBP of $25 \mathrm{mmHg}$ ) [35]. Guidelines also recommend avoidance of PDE5 inhibitors within 24 hours of administration of long-acting nitrates, and avoidance of nitrates until 24 hours or 48 hours after use of sildenafil or tadalafil, respectively (a suitable time interval after vardenafil use has not yet been determined) [35]. Avanafil was approved for use in April 2012, so inclusion was not considered when these guidelines were developed. However, concomitant use of avanafil and nitrate is also strictly contradicted if the doses are $<12$ hours apart [7]. In patients with ED who have stable coronary artery disease, data have shown that for some patients, oral nitrates may be safely discontinued during continued treatment with beta-blockers or calcium antagonists [36].

\section{Study limitations}

All study drugs (avanafil, sildenafil, and placebo) were over-encapsulated to assure blinding and comparability. Although overencapsulation is a well-recognized method for accomplishing blinding and comparability, excipients placed inside the capsule for this process may affect the dissolution profile of a drug. A recent in vivo study comparing intravenous and over-encapsulated moxifloxacin, however, demonstrated that over-encapsulation neither altered the peak or total systemic exposure nor the pharmacodynamics of the drug, after oral administration [37]. Another limitation is that this clinical trial comprised healthy, normal volunteers, and results may be different in populations with known vascular disease (especially those using other concurrent pharmacotherapy). For example, beta-blockers may augment the BP-lowering effect of PDE5 inhibitors (although the effect is minor), which may further increase vulnerability to the BPlowering effect of nitrates [38]. Finally, tolerance, tachyphylaxis, or adaptive mechanisms may present different results for chronic users of PDE5 inhibitors and/or NTG.

\section{Conclusions}

The results of this Phase I study suggest that a PDE inhibitor with high specificity for inhibiting PDE5 compared with other PDE isoforms, along with a short half-life, may potentiate the hemodynamic (i.e., BP and HR) effects of NTG to a lesser degree than other agents in this class that are less specific for PDE5, and/or longer acting.

\section{Future perspectives}

Although the data presented here are preliminary, the information may be helpful for several clinical situations. Examples include: an Emergency Room physician faced with an acute management decision (e.g., angina) for a patient who has recently used a PDE5 inhibitor; or a Primary Care physician who needs to discuss ED options with a patient who uses nitrates. If applicable, these patients may be provided with options to replace 
their nitrates with another agent, such as a beta-blocker [39]. Furthermore, the data obtained in the present study will hopefully serve as a stimulus to drive further research that may clarify a deeper understanding of drug-drug pharmacodynamic interactions with these two classes of medications.

\section{Acknowledgements and funding declaration}

The study was designed and funded by VIVUS, Inc., who also wrote the study protocol and funded the data analyses. This was a single-center study conducted at Celerion (formerly MDS Pharma Services) in Tempe, AZ, USA. Celerion received funds from VIVUS, Inc., for their participation in the study. BlueMomentum (a division of the KnowledgePoint360 Group) provided writing, editorial, and graphics support for the development of this manuscript; their participation was funded by VIVUS, Inc.

\section{Author contributions}

D. Swearingen was involved in the data acquisition, drafting and critical revision of the manuscript, and supervision. A. Nehra was involved in the analyses and interpretation of the data, drafting and critical revision of the manuscript, and supervision. S. Morelos was involved in data acquisition, drafting and critical revision of the manuscript, and supervision. C. A. Peterson was involved in the conception and design of the study, acquisition, analyses and interpretation of the data, drafting and critical revision of the manuscript, and supervision.

\section{References}

1. Selvin E, Burnett AL, Platz EA. Prevalence and risk factors for erectile dysfunction in the US. Am J Med 2007;120:151-7.

2. Aytac IA, McKinlay JB, Krane RJ. The likely worldwide increase in erectile dysfunction between 1995 and 2025 and some possible policy consequences. BJU Int 1999;84:50-6.

3. Thompson IM, Tangen CM, Goodman PJ, Probstfield, JL, Moinpour CM, Coltman CA. Erectile dysfunction and subsequent cardiovascular disease. JAMA 2005;294:2996-3002.

4. Viagra (sildenafil citrate) tablets [prescribing information]. Pfizer Inc; New York, NY; 2010.

5. Levitra (vardenafil hydrochloride) tablets [prescribing information]. Bayer HealthCare Pharmaceuticals, Inc; Wayne, NJ; 2011.

6. Cialis (tadalafil) tablets [prescribing information]. Eli Lilly \& Company; Indianapolis, IN; 2011.

7. Stendra (avanafil) tablets [prescribing information]. VIVUS, Inc; Mountain View, CA; 2012.

8. Schwartz BG, Kloner RA. Drug interactions with phosphodiesterase-5 inhibitors used for the treatment of erectile dysfunction or pulmonary hypertension. Circulation 2010;122:88-95

9. Bender AT, Beavo JA. Cyclic nucleotide phosphodiesterases: molecular regulation to clinical use. Pharmacol Rev 2006;58:488-520.
10. Revatio (sildenafil) tablets [prescribing information]. Pfizer, Inc; New York, NY; 2012.

11. Adcirca (tadalafil) tablets [prescribing information]. Eli Lilly \& Company; Indianapolis, IN; 2012.

12. Araujo AB, Hall SA, Ganz P et al. Does erectile dysfunction contribute to cardiovascular disease risk prediction beyond the Framingham risk score? J Am Coll Cardiol 2010;55:350-6.

13. Batty GD, Li Q, Czernichow $S$ et al. Erectile dysfunction and later cardiovascular disease in men with type 2 diabetes: prospective cohort study based on the ADVANCE (Action in Diabetes and Vascular Disease: Preterax and Diamicron Modified-Release Controlled Evaluation) trial. J Am Coll Cardiol 2010;56:1908-13.

14. Navaneethan SD, Vecchio M, Johnson DW et al. Prevalence and correlates of self-reported sexual dysfunction in CKD: a meta-analysis of observational studies. Am J Kidney Dis 2010;56:670-85.

15. Seftel AD, Sun P, Swindle R. The prevalence of hypertension, hyperlipidemia, diabetes mellitus and depression in men with erectile dysfunction. J Urol 2004;171 (6 Pt 1):2341-5.

16. Burchardt M, Burchardt T, Baer L et al. Hypertension is associated with severe erectile dysfunction. J Urol 2000;164:1188-91.

17. Chew KK, Finn J, Stuckey B et al. Erectile dysfunction as a predictor for subsequent atherosclerotic cardiovascular events: findings from a linked-data study. J Sex Med 2010;7(1 Pt 1):192-202.

18. Eaton CB, Liu YL, Mittleman MA, Miner M, Glasser DB, Rimm EB. A retrospective study of the relationship between biomarkers of atherosclerosis and erectile dysfunction in 988 men. Int J Impot Res 2007;19:218-25.

19. Fung MM, Bettencourt R, Barrett-Connor E. Heart disease risk factors predict erectile dysfunction 25 years later: the Rancho Bernardo Study. J Am Coll Cardiol 2004;43: 1405-11.

20. Mulhall J, Teloken P, Brock G, Kim E. Obesity, dyslipidemias and erectile dysfunction: a report of a subcommittee of the Sexual Medicine Society of North America. J Sex Med 2006;3:778-86.

21. Riedner CE, Rhoden EL, Ribeiro EP, Fuchs SC. Central obesity is an independent predictor of erectile dysfunction in older men. J Urol 2006;176(4 Pt 1):1519-23.

22. Billups KL, Bank AJ, Padma-Nathan H, Katz S, Williams R. Erectile dysfunction is a marker for cardiovascular disease: results of the minority health institute expert advisory panel. J Sex Med 2005;2:40-50; discussion: 50-2.

23. Inman BA, Sauver JL, Jacobson DJ et al. A populationbased, longitudinal study of erectile dysfunction and future coronary artery disease. Mayo Clin Proc 2009;84:108-13.

24. Ma RC, So WY, Yang X et al. Erectile dysfunction predicts coronary artery disease in type 2 diabetes. J Am Coll Cardiol 2008;51:2045-50.

25. Salem S, Abdi S, Mehrsai A et al. Erectile dysfunction severity as a risk predictor for coronary artery disease. J Sex Med 2009;6:3425-32. 
26. Nehra A. Erectile dysfunction and cardiovascular disease: efficacy and safety of phosphodiesterase type 5 inhibitors in men with both conditions. Mayo Clin Proc 2009;84: 139-48.

27. Dong JY, Zhang YH, Qin LQ. Erectile dysfunction and risk of cardiovascular disease: meta-analysis of prospective cohort studies. J Am Coll Cardiol 2011;58:1378-85.

28. Bitar F, Akhter MW, Khan S, Singh H, Elkayam U. Survey of the use of organic nitrates for the treatment of chronic congestive heart failure in the United States. Am J Cardiol 2004;94:1465-8.

29. Cheitlin MD, Hutter AM Jr, Brindis RG et al. ACC/AHA expert consensus document. Use of sildenafil (Viagra) in patients with cardiovascular disease: American College of Cardiology/American Heart Association. J Am Coll Cardiol 1999;33:273-82.

30. Kotera J, Mochida $\mathrm{H}$, Inoue $\mathrm{H}$ et al. Avanafil, a potent and highly selective phosphodiesterase-5 inhibitor for erectile dysfunction. J Urol 2012;188:668-74.

31. Wang R, Burnett AL, Heller W et al. Selectivity of avanafil, a PDE5 Inhibitor for the treatment of erectile dysfunction: implications for clinical safety and tolerability. J Sex Med 2012;9:2122-9.

32. Allison M, Grant T, Obaidi M, Marenco T, Yee S, Day WW. Pharmacokinetics of avanafil, a novel, rapidly absorbed, selective PDE5 inhibitor for the treatment of mild to severe erectile dysfunction. J Sex Med 2011;8:S466-7.

33. International Conference on Harmonisation. International Conference on Harmonisation of Technical Requirements for Registration of Pharmaceuticals for Human Use [International Conference on Harmonisation website]. Available at: http://www.ich.org/products/guidelines.html. [Last accessed 1 February 2013].
34. World Medical Association General Assembly. WMA Declaration of Helsinki-Ethical Principles for Medical Research Involving Human Subjects [World Medical Association website]. Available at: http://www.wma.net/ en/30publications/10policies/b3/. [Last accessed 1 February 2013].

35. Fihn SD, Gardin JM, Abrams J, et al. 2012 ACCF/AHA/ ACP/AATS/PCNA/SCAI/STS Guideline for the Diagnosis and Management of Patients With Stable Ischemic Heart Disease: a Report of the American College of Cardiology Foundation/American Heart Association Task Force on Practice Guidelines, and the American College of Physicians, American Association for Thoracic Surgery, Preventive Cardiovascular Nurses Association, Society for Cardiovascular Angiography and Interventions, and Society of Thoracic Surgeons. Circulation 2012;126:e354-e471.

36. Jackson G, Martin E, McGing E, Cooper A. Successful withdrawal of oral long-acting nitrates to facilitate phosphodiesterase type 5 inhibitor use in stable coronary disease patients with erectile dysfunction. J Sex Med 2005;2:513-16.

37. Mason JW, Florian JA Jr, Garnett CE, Moon TE, Selness DS, Spaulding RR. Pharmacokinetics and pharmacodynamics of three moxifloxacin dosage forms: implications for blinding in active-controlled cardiac repolarization studies. J Clin Pharmacol 2010;50:1249-59.

38. Zusman RM, Prisant LM, Brown MJ. Effect of sildenafil citrate on blood pressure and heart rate in men with erectile dysfunction taking concomitant antihypertensive medication. Sildenafil Study Group. J Hypertens 2000;18:1865-9.

39. Nehra A, Jackson G, Miner M, et al. The Princeton III Consensus recommendations for the management of erectile dysfunction and cardiovascular disease. Mayo Clin Proc 2012;87:766-78. 